\title{
A Robust Multi-Factor Recommender System for Online Libraries and E-book Portals
}

\author{
Disha Sharma ${ }^{1}$, Sumit Kaur ${ }^{2}$ \\ ${ }^{1}$ Chandigarh University \\ ${ }^{2}$ Assistant Professor, Department of CSE, Chandigarh University
}

\begin{abstract}
The online libraries or book portals have gained significant popularity among the readers in the recent years. The online libraries or book portals receive a number of users and are programmed to show the popular books among similar reader and user profile (collaborative filtering) and the books similar to the reading history of reader (content based filtering). These methods are incorporated to estimate the appropriate suggestions, recommendations and new arrivals according to user interests and history. The recommender system calculates the recommendations on the basis of various online and local features which include the e-book/book popularity, user rating, trust factor, page rank, total access, etc. The recommendations are calculate for the user convenience to find the related stuff easily over the populated book libraries and e-book shopping portals. In this paper, the entire has been based upon solving the problem of correct recommendations according to the user's interest, profile contents and similar user's history and interests. The proposed model will be evaluating the global factors such as Alexa Recommendation, Google page rank and trust factor for appropriate recommendations. The local factors of total access, rating and downloads or purchases, number of readers and user opinion will be exercised to calculate the correct recommendations. The major area of concern will be the institutional online libraries and shared libraries such as Google Books. The expected results would be collected in the form of accuracy, precision, recall and F1-error.
\end{abstract}

Keywords: Expert systems, Book Recommender System, recommendation algorithm, library suggestions engine

\section{Introduction}

Recommendation system defines a position of an book or ebook in a list by comparing the book with other books such that preeminent book will have higher rank and the inferior book will have given lower rank. The Recommendation system helps the readers to decide which option to be select from multiple options. With the increasing use of internet, the use of online websites for the purchase and sales such as apparels, books, videos, movies, songs, furniture, etc has also increased. So, Recommendation system will help readers to choose best sites or e-book among various options. The Recommendation is assigned by using multiple factors. The different ways to assign a rank are:

Standard competition Recommendation: In this, the books are compared and similar books will have the same rank. The gap is left after the similar books. So another book should given a rank according to number of books above it plus one. For example, "1 23557 Recommendation"

Modified competition Recommendation: This strategy is similar to the standard competition Recommendation but the gap is considered prior to the same Recommendation. For example,"1234667"

Dense Recommendation: In dense Recommendation, the gap is not considered. The ranks are granted in a proper sequence i.e. one plus the book ranked before it. The similar book will have same rank. For example,"1 2345567 ”.

Ordinal Recommendation: In this, every book is given different rank number including the equal books when compared. The equal books are ranked by following some criteria. For example, "1 234567 ",

Fractional Recommendation: In fractional Recommendation, the books that are equal after comparing will have same rank in Recommendation system but rank will be average of the book that are equal after comparing. For example,"1 2.52 .5456 ".

Online Libraries stands for Electronic commerces. It is the buying and selling of goods and services using a network such as internet. Online Libraries is used in online shopping, bill payments, ebooks, e-mail, etc. The companies provide these services are Amazon, flipkart, quikr, snapdeal, olacabs and paytm. Online Libraries is mainly used because it reduces transaction cost and improve level of reader services. It also has worldwide market accessibility. Various categories of Online Libraries are: business-to-business, business-to-reader, reader-to-reader or reader-to-business.

The Recommendation system is very beneficial especially in Online Libraries. Some of the benefits of Recommendation are:

- It helps the readers to decide which option to be chosen among the multiple options and in less amount of time.

- The personalized Online Libraries Recommendation system has increased the trust and loyalty relationship between readers and website owners.

- Provides comparison between different companies and websites which increase competition between the website owners which results in better and improved services.

Recommendation e-book is a process to rank various ebooks. Firstly a single e-book is ranked then grouping of ebooks are performed and the groups of e-book is ranked. For example let's take a e-book Mobile phones, they there are grouped according to their companies like Samsung, Nokia, Apple, HTC etc. and they are sub grouped according to their models and at each step Recommendation is performed.

Semantic web is used to store data in the computer system without the guidance of human and can be easily readable to humans in form of web pages. In semantic web data stores are created on web. It develops the common framework that 


\section{International Journal of Science and Research (IJSR) \\ ISSN (Online): 2319-7064}

Index Copernicus Value (2013): 6.14 | Impact Factor (2015): 6.391

permits to share the data among companies, communities or applications. The semantic web is amalgamated with the Recommendation system and in this dynamic amalgamation is performed. In dynamic amalgamation, if a new e-book is entered in a ranked list then, it will automatically generate the rank of the e-book by comparing it with other e-books in the list and after that it updates the Recommendation.

\section{What To Include In Multiple Factors}

In Recommendation system, multiple factors are considered to rank a website or a e-book. The following factors will be used for the Recommendation system evaluation:

- Alexa Rank: Alexa rank the website rank calculated by the Alexa website. The Alexa website tracks the users on user location, pageviews per users, etc.

- Google Page Rank: Google Page Rank (PR) is another website Recommendation model by the Google Inc. The Google PR is measured on the scale of 0 to 10 and includes the quality backlinks to the page or website.

- Alexa Trust Rank: The Alexa trust is calculated by the data provided by the Alexa Rank. The trust factor evaluates the bounce rate, daily Perviews per visitor, Daily time on site, etc. The trust factor gives the reliability of the websites for its users.

- Google Trust Factor: Google's trust factor is a combination of many factors that they use to apply a value of how trustful a site is. The more trustful a site is seen the more likely its articles will be ranked higher on specific Google searches. Some factors that make a site less trustful are that its seen as a harmful and/or has low quality content.

\section{Literature Review}

Neha verma et.al. have created an algorithm named the "SNEC page Recommendation algorithm" which is the. semantic and neural based Online Libraries page Recommendation algorithm. The website has been rank by using the various appropriate features to help the reader to pick the best e-book among multiple e-books. By this algorithm, the companies can known about their weakness and provide the reader with improvised e-book. The results provide much accurate results but the drawback of this algorithm is that it includes less number of features.

Hepp, Martin et. al. has worked for the Online Libraries particularly schema.org and GoodRelations for researchers and practitioners on the web of the data. In the paper, the author has given an introduction and primary guidance on the conceptual structure of schema.org. they have created the patterns for demand and ownership which include the variety of books like furniture, apparels, electronics devices, cosmetics, books, etc. and have created a full tool chain for producing and consuming the particular data. The author have also discussed the topic like authentication (e.g. with WebID), identity, access control; data management issues from the publisher and reader perspective and micropayment services. The drawbacks of this application is restricted to micro-data which is not applicable for Online Libraries ebook Recommendation system.
Sessoms, Matthew, and Kemafor Anyanwu has worked model and algorithms for enabling a Package Query criterion on the Semantic Web. The package query is the combination of multiple queries that helps to get resource combination on a semantic web. The subclass of such queries is "skyline package queries". In contrast to package queries on a single relational models, the RDF model have injected the challenge of determining the skyline package of ternary relations over multiple joins. The different combination of new operators for skyline package queries relational query operators and RDF data storage models have developed the four strategies for evaluation. The author lacks in the use of additional techniques for optimization such as prefetching as well as the integration of top-k techniques.

Malhotra, Dhairya et. al has used the back propagation neural network on Intelligent web mining to upgrade web page rank. Because of the increase in information resources, the web is developing at faster rate. But its huge size increases the difficulties during the research process in extracting the required information from web. This problem can be overcome by using the personalized web search but the user has to give his personal information to maintain privacy. In the paper, the author addresses all the above mentioned issues by using the back propagation neural network for implementing page Recommendation process.

Mital, Monika et. al. have proposed an integrative framework in the context of e-procurement and ERP to identify determinant of choice for SaaS. In this paper, the author has tried to classify, identify and rank the dimensions which are influencing SaaS sourcing decision. Using extended AHP (analytic hierarchy process) method, the framework is analyzed that helped in identifying quality and costs and then weights criteria are identified using the data which was collected by 8 users and 9 service providers of SaaS based on ERP and e-procurement.

\section{Problem Formulation}

The word "page or e-book range" means to rank the system to display the results and to provide suggestions to others. In day to day activities we rely on Online Library Recommendation system such as the page indexing or ebook Recommendation of movies like reviews of movies from newspaper etc These Online Library Recommendation basically help us to decide what we should opt for when we have multiple options An Online Library Recommendation system (OLRS) helps people that have not sufficient personal experience or competence to evaluate the, potentially overwhelming, number of alternatives offered by a Web site. [1, 2] In their simplest form RSs recommend to their users personalized and ranked lists of books Provide readers with information to help them decide which books to purchase. The existing model does not consider the page loading speed comparison, ease of navigation comparison, page availability options (online/offline) and security comparison of the online resources on the Online Libraries websites. [1-3] The Online Libraries website Recommendation model by using the latter parameters can be used to determine the correct possible result for the Online Libraries Recommendation. The 


\section{International Journal of Science and Research (IJSR) \\ ISSN (Online): 2319-7064}

Index Copernicus Value (2013): 6.14 | Impact Factor (2015): 6.391

keyword analysis system to clearly choose the available groups of Online Libraries pages for the effective Recommendation system. [1] The existing model is not capable of producing the real-time web priority listings, which can be further improved by using the page load speeds, navigation accessibility and ease, online/offline comparison, security comparison etc as per stated in the future work. The big data analytical techniques can also be incorporated to fulfill the need to speeded up Online Libraries Recommendation to reduce the overhead and overburdened query timings. [3-4]

\section{Methodology}

During the very first step, the resource representation would be performed using the various types of algorithms. The system obtains an internal representation of the resources based on their scopes. The system obtains an internal representation of the dataset based on their preferred research resources and topics of interest. The system generates the Online Libraries Recommendation system according to the hybrid filtering approach. From the preference matrix about research resources, the system obtains a quality score for each book. The system aggregates the estimated relevance of a research resource and its quality score in a single score. The users provide the system their opinion about the received Online Libraries Recommendations.

\section{Conclusion}

The proposed model design has been undergone the in-depth evaluation for its workflow model and design. The proposed model has been designed to incorporate the higher accuracy in the Recommendation model over the recommendations and suggestions generated by calculating the several parameters to evaluate the e-books listed over the Online Libraries portals. The proposed model has been designed to utilize the Google Page Rank model, Alexa Website Recommendation, Alexa Trust and several other offline or local factors to evaluate the actual rank of the e-book and page in the Online Libraries Recommendation system. The proposed model is expected to solve the major shortcomings of the existing models to make the user experience much better than the previous models.

\section{References}

[1] Verma, Neha, Dheeraj Malhotra, Monica Malhotra, and Jatinder Singh. "Online Libraries Website Recommendation Using Semantic Web Mining and Neural Computing." Procedia Computer Science 45 (2015): pp. 42-51, ELSEVIER.

[2] Hepp, Martin. "The Web of Data for Online Libraries: Schema. org and GoodRelations for Researchers and Practitioners." In Engineering the Web in the Big Data Era, pp. 723-727. Springer International Publishing, 2015.

[3] Sessoms, Matthew, and Kemafor Anyanwu. "Enabling a Package Query Paradigm on the Semantic Web: Model and Algorithms." In Transactions on Large-
Scale Data-and Knowledge-Centered Systems XIII, pp. 1-32. Springer Berlin Heidelberg, 2014.

[4] Malhotra, Dhairya. "Intelligent web mining to ameliorate Web Page Rank using Back-Propagation neural network." In Confluence The Next Generation Information Technology Summit (Confluence), 2014 5th International Conference-, pp. 77-81. IEEE, 2014.

[5] Furukawa, Takao, Kaoru Mori, Kazuma Arino, Kazuhiro Hayashi, and Nobuyuki Shirakawa. "Identifying the evolutionary process of emerging technologies: A chronological network analysis of World Wide Web conference sessions." Technological Forecasting and Social Change 91 (2015): 280-294.

[6] Scioscia, Floriano, Michele Ruta, Giuseppe Loseto, Filippo Gramegna, Saverio Ieva, Agnese Pinto, and Eugenio Di Sciascio. "A Mobile Matchmaker for the Ubiquitous Semantic Web." International Journal on Semantic Web and Information Systems (IJSWIS) 10, no. 4 (2014): 77-100.

[7] Mital, Monika, Ashis Pani, and Ram Ramesh "Determinants of choice of semantic web based Software as a Service: An integrative framework in the context of e-procurement and ERP." Computers in Industry 65, no. 5 (2014): 821-827.

[8] D.T. Green and J. M. Pearson, "The examination of two web site usability instruments for use in B2C Online Libraries organizations," Journal of Computer Information Systems, Vol. 49, No. 4, 2009, pp. 19-32

[9] T. Wang and Y. Lin, "Accurately predicting the success of B2B ecommerce in small and medium enterprises," Expert Systems with Applications, Vol. 36, No. 2, published by Elsevier, 2009, pp. 2750-2758.

[10] M. Lazarica and I. Lungu, Aspecte privind proiectarea sistemelor de comert electronic, ASE Publishing House, 2007, pp. 147. 